93 PALLIATIVE MEDICINE DOCTOR AND PARAMEDIC JOIN TO FORM A PALLIATIVE CARE RAPID RESPONSE CAR. A PILOT STUDY

Edward Presswood, Ed O'Brian, Jo Hayes, Idris Baker, Nikki Pease. Wales Deanery, Welsh Ambulance Service NHS Trust, Marie Curie Hospice Cardiff and Vale, Ty Olwen, Velindre Cancer Centre

10.1136/bmjspcare-2019-ASP.116

Aim To pilot a palliative medicine doctor and paramedic working together within the community to respond to urgent '999' calls. Is the concept feasible, beneficial to patients and cost effective?

Method Four palliative medicine doctors across South Wales partnered the End of Life Care Lead Paramedic for WAST (EO'B) to form a PCRRC. Potential patients were identified from the list of contemporaneous logged calls for paramedics to respond to. The PCRRC responded to any calls where it seemed likely that it could have a positive impact upon the care of patients.

Result During the four pilot shifts the PCRRC attended four calls and gave telephone advice to three calls. In total 21 hours of doctor time was spent 'on the road'. The anecdotal feedback from the four doctors is mixed. There was not felt to be an overwhelming need for the service but on two occasions it did have an impact upon decision making, including two decisions not to admit patients. The experience improved doctors' insight into paramedic care of patients with palliative care needs.

Conclusion This is a small feasibility study with inherent biases. The PCRRC concept is feasible and can benefit acute clinical decision making but this pilot suggests that it is unlikely to be an efficient use of resources. There are benefits of the PCRRC model for learning, co-ordination of care, and facilitating shared decision making. We are considering other interventions to improve the interaction between palliative care teams and WAST.

\section{KEY INFORMATION SUMMARY (KIS) GENERATION FOR PEOPLE WHO DIED IN SCOTLAND IN 2017: A MIXED METHODS STUDY}

Anne Finucane, Deborah Davydaitis, Zoe Horseman, Emma Carduff, Julia Tapsfield, Paul Baughan, Richard Meade, Tim Warren, Juliet Spiller, lan Thompson, Kirsty Boyd, Scott A Murray. Marie Curie Hospice Edinburgh, University of Edinburgh, Marie Curie Hospice Glasgow, NHS Lothian, Healthcare Improvement Scotland, Marie Curie, Scottish Government

\subsection{6/bmjspcare-2019-ASP.117}

Introduction Key Information Summaries (KIS), introduced throughout Scotland in 2013, are shared electronic clinical summaries used to guide urgent care in the community and emergency hospital admission. The percentage of people with a KIS at the time of death can act as an indicator of access to palliative care.

Aims

1. To estimate the extent of KIS generation for people who die with an advanced progressive condition.

2. To explore GP perspectives of commencing and updating a KIS.

Methods A retrospective review of the electronic records of all patients who died in 18 Scottish general practices in 2017; semi-structured interviews with one healthcare professional in each practice.

Results Data on 1304 decedents was collected (49\% female, $51 \%$ male). Of these, 1034 had an advanced progressive condition at the time of death. $69 \%$ died with a KIS. This was highest for patients with cancer $(80 \%)$. Of the 712 patients with a KIS, 69\% $(n=488)$ had resuscitation status recorded. $60 \%$ had a next of kin name documented in the KIS. 52\% of KISs were highly useful (clear plan regarding patient wishes concerning current care and future planning), $45 \%$ were useful. $37 \%$ of patients with a KIS died in hospital, compared with $65 \%$ without a KIS. The special notes section was considered the most useful part of the KIS.

Conclusion The proportion of patients with an advanced progressive condition who have a KIS at the time of death has increased from 60\% in 2014 (Tapsfield et al 2016) to 69\% in 2017. KISs are nearly always perceived as useful or highly useful, and are associated with a higher likelihood of dying in a community setting. Better information regarding next of kin/ carers would further improve KIS quality, and a renewed focus on KIS generation for people with organ failure is warranted.

\section{IMPLEMENTING ELECTRONIC DISCHARGES AT A NON- NHS HOSPICE: THE BENEFITS AND MANY CHALLENGES}

Siwan Seaman. Marie Curie Hospice Cardiff and the Vale

\subsection{6/bmjspcare-2019-ASP.118}

Aims The aim of the eDischarge project was to share discharge advice letters (DALs) with all relevant healthcare professionals at the point of discharge. The existing process was for letters to be dictated, typed, corrected by a clinician, amended by admin and sent in the post.

Methods This quality improvement project used improving quality together (IQT) methodology. The measure chosen to monitor progress in reaching the aim was the time between discharge from the hospice and the DAL being sent to the GP. The ward secretary held a database of all discharge dates and completion dates of DALs which allowed us to measure the times taken as a weekly average to plot on a run chart. A pilot of MTeD (the electronic discharge prescription and DAL programme used within the Welsh NHS Clinical Portal in use across all Health Boards in Wales) occurred during 2 weeks in June 2018.

Results Prior to the pilot the mean time from discharge to letter being posted over a 10 week baseline period of data collection was 6.9 days. During the 2 week pilot this average dropped to 1.5 days. In addition, following the point of release the electronic version was reaching GPs within 4 hours of discharge as opposed to being dependent on variable postal times.

Discussion and conclusion The results of the pilot are indisputable with significant reductions in the delay in sharing medication and clinical information with key professionals and provide the added benefit of being available on the NHS clinical portal for other healthcare professionals to access e.g. if the patient attends $\mathrm{A}$ and $\mathrm{E}$ the day after discharge. However, many challenges relating to use of the relevant NHS clinical applications and IT infrastructure in a third sector setting needed to be overcome. 